\title{
Clinical, radiological and biological features of lung metastases in gastrointestinal stromal tumors (Case reports)
}

\author{
MARGHERITA NANNINI ${ }^{1}$, GUIDO BIASCO ${ }^{1,6}$, VALERIO DI SCIOSCIO ${ }^{2}$, MONICA DI BATTISTA ${ }^{1}$, \\ MAURIZIO ZOMPATORI ${ }^{2}$, FAUSTO CATENA ${ }^{3}$, PAOLO CASTELLUCCI ${ }^{4}$, \\ PAOLA PATERINI ${ }^{6}$, ANGELO PAOLO DEI TOS ${ }^{7}$, FRANCO STELLA ${ }^{5}$, \\ ALESSANDRA MALEDDU ${ }^{1}$ and MARIA ABBONDANZA PANTALEO ${ }^{1,6}$
}

\author{
${ }^{1}$ Department of Hematology and Oncological Sciences 'L.A. Seragnoli', ${ }^{2}$ Division of Pneumo-Nefro, Department of \\ Radiology, Departments of ${ }^{3}$ Transplant, General and Emergency Surgery, ${ }^{4}$ Nuclear Medicine and ${ }^{5}$ Thoracic Surgery, \\ S. Orsola-Malpighi Hospital, ${ }^{6}$ Interdepartmental Centre of Cancer Research 'G. Prodi', University of Bologna, Bologna; \\ ${ }^{7}$ Departments of Pathology and Oncology, General Hospital of Treviso, Treviso, Italy
}

Received May 10, 2010; Accepted October 7, 2010

DOI: 10.3892/or_00001049

\begin{abstract}
Gastrointestinal stromal tumors (GISTs) are mesenchymal tumors that most frequently arise in the gastrointestinal tract. The liver and peritoneum are common sites of distant GIST lesions, whereas lung metastases are infrequent, accounting for $7 \%$ of all lesions. The clinical significance of these metastases remains unknown. Although lung metastases are relatively rare in the natural history of GIST, they may become more prevalent due to increased patient life expectancy. The present report describes four patients with GIST who had lung metastases. Two were female (54 and 28 years of age), and two were male (64 and 44 years of age). The primary GISTs were localized in the stomach in two patients and in the small intestine in the other two patients. Three patients presented with multiple lung lesions and one presented with one lung lesion. Lung metastases were present at the time of initial diagnosis in one patients, and were observed during the follow-up period in the other three. In this report we detail the clinical presentation and radiological features of the lung lesions as observed by computed tomography (CT) and computed tomography/ positron emission tomography (CT/PET). We describe each patient's clinical history and treatment which included surgery and the tyrosine kinase inhibitors, imatinib and sunitinib, and the novel therapy, nilotinib. Moreover, we discuss some biological aspects of this relatively rare occurrence and the resulting clinical implications. These findings may help
\end{abstract}

Correspondence to: Dr Margherita Nannini, University of Bologna, Department of Hematology and Oncological Sciences 'L.A. Seragnoli', S. Orsola-Malpighi Hospital, Via Massarenti 9, I-40138 Bologna, Italy

E-mail: maggie.nannini@gmail.com

Key words: gastrointestinal stromal tumor, lung metastases, computed tomography, computed tomography/positron emission tomography clinicians to manage lung metastases arising from GISTs in future.

\section{Introduction}

Gastrointestinal stromal tumors (GISTs) are common mesenchymal tumors in the gastrointestinal tract which arise from the interstitial cells of Cajal (ICC). Specific abnormalities of the KIT and PDGFR- $\alpha$ (PGDFRA) receptors are the main oncogenic event in GISTs, and these receptors remain the most relevant therapeutic targets (1). Approximately 75$80 \%$ of GISTs harbor gain-of-function c-KIT mutations, 7$10 \%$ harbor gain-of-function PGDFRA mutations, and the remaining are defined as wild-type (WT) because they have no KIT or PDGFRA mutations $(2,3)$. The mutational status of these genes affect disease progression and response to tyrosine kinase inhibitors $(4,5)$. The liver and peritoneum are the most frequent sites of distant metastases from GISTs. but lung lesions are rare, comprising $7 \%$ of these metastases (6). To date, lung metastases have been reported as anecdotal findings, and few studies have examined the clinical significance of these lesions (7-12). We describe four patients with GIST treated at our institution who presented with lung metastases, focusing on the clinical, radiological and biological features of these lesions.

\section{Case reports}

Case 1. In 2003, a 64-year-old male underwent gastric resection for a high-risk GIST harboring a homozygous c-KIT deletion mutation (aa 550-555I, KPMYEV) in exon 11. In 2008, a follow-up CT scan revealed two lung lesions and a solid opaque region $14 \mathrm{~mm}$ in diameter in the parenchyma of the right lower lobe, which was confirmed by CT/PET (Fig. 1A and B). After 15 months of treatment with $400 \mathrm{mg}$ daily imatinib (Glivec ${ }^{\circledast} /$ Gleevec $^{\circledR}$, Novartis Pharmaceuticals Corp., East Hanover, NJ, USA), the patient achieved a complete metabolic response by PET and a partial response by $\mathrm{CT}$ of the lung lesion (7.8 vs. $14 \mathrm{~mm}$ ) (Fig. 1C and D). He 
A

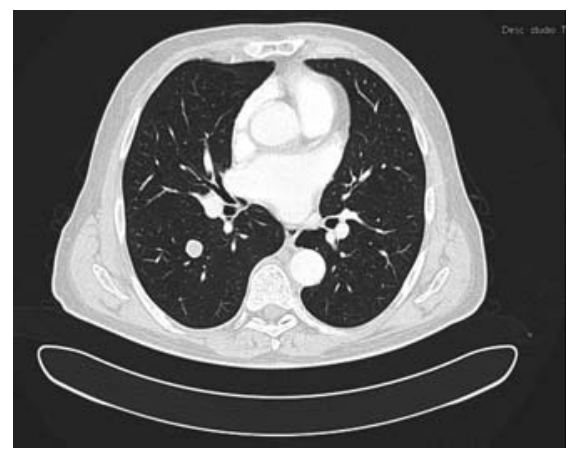

B
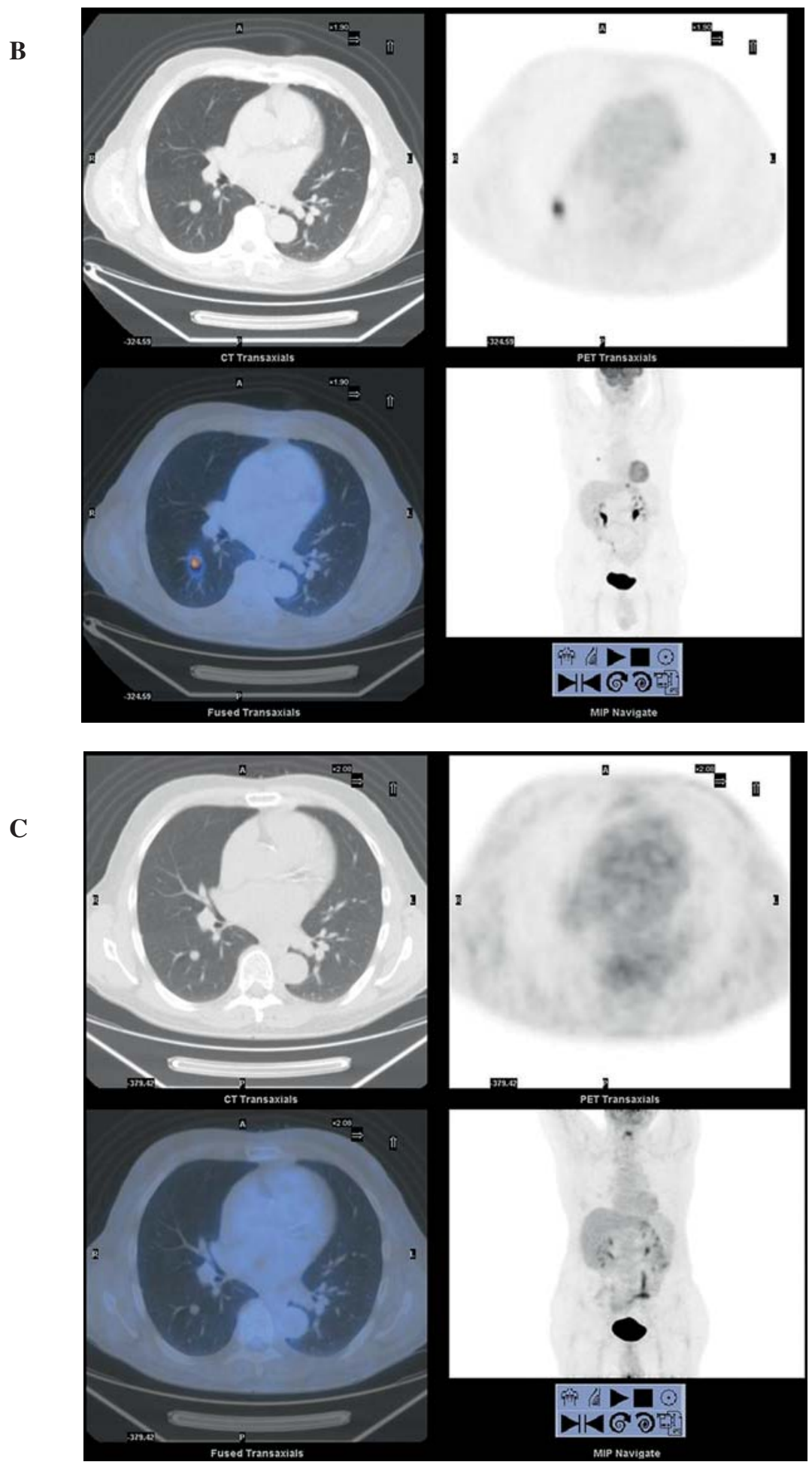

Figure 1. A, B and C. Computed tomography. 


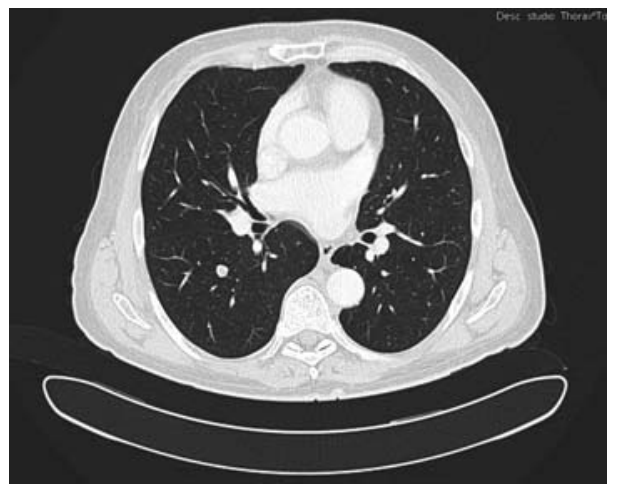

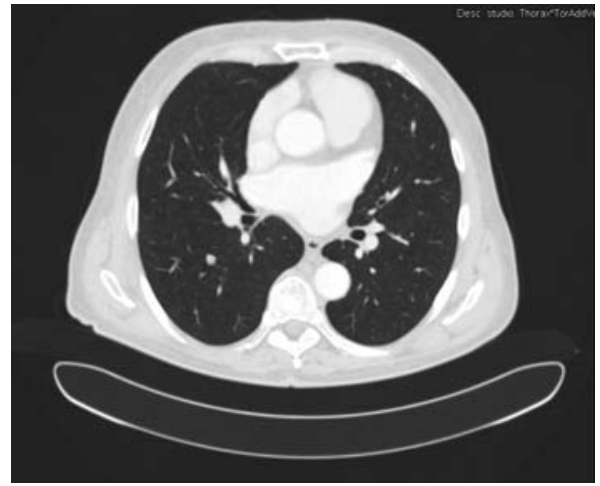

Figure 1. (A) Computed tomography (CT) taken in August 2008 revealed a 14-mm solid opaque region in the parenchyma of the lower right lobe of the lung. (B) Computed tomography/positron emission tomography (CT/PET) evaluation in September 2008 confirmed the presence of an ipermetabolic nodule in the lower region of the right lobe of the lung. (C) October 2008: early CT/PET taken after one month of imatinib treatment showed a complete metabolic response. (D) December 2008: CT evaluation after three months of treatment showed a partial response, with a reduction in lesion diameter (7.8 vs. 14 mm). (E) CT taken at the most recent evaluation (December 2009) showed stable disease.

A

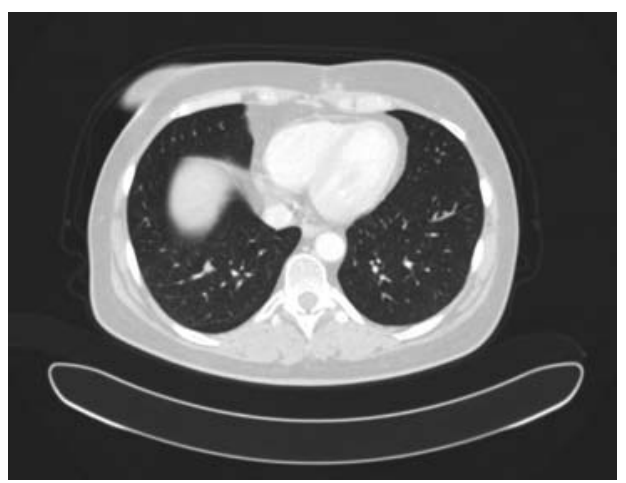

$\mathbf{C}$

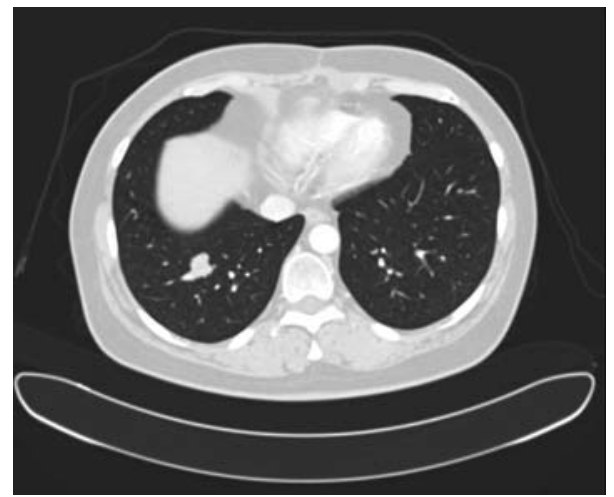

B

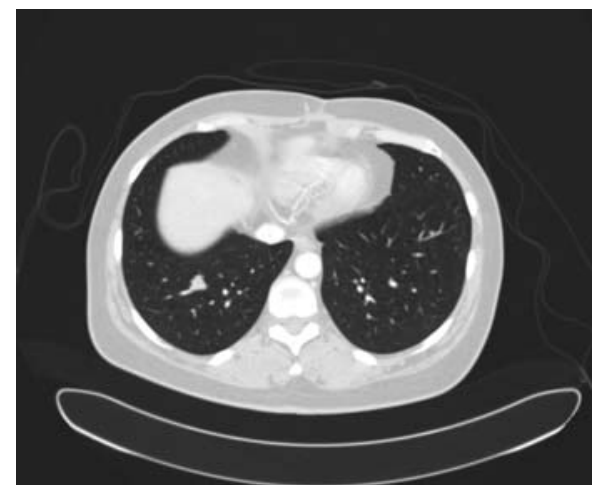

D

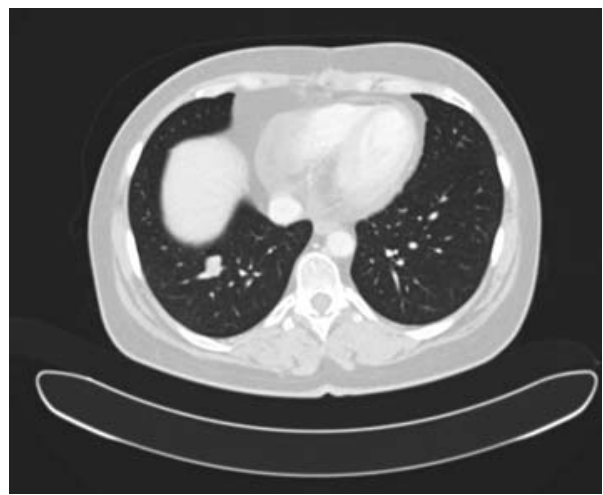

Figure 2. (A) February 2009: a nodular image at the right low lobe was observed, but was not suspected to be a lung lesion because of the small tumor diameter $(5 \mathrm{~mm})$. (B) July 2009: a mild dimensional increase of the known lung nodule was observed (9 vs. $5 \mathrm{~mm}$ ). (C) November 2009: an additional increase in the lung lesion diameter was observed (13 vs. $9 \mathrm{~mm}$ ). (D) February 2010: at the last computed tomography evaluation, the patient was found to have stable disease.

continued on imatinib and his most recent $\mathrm{CT}$ evaluation showed stable disease (Fig. 1E).

Case 2. In 2004, a 54-year-old female underwent ileal resection for a high-risk ileal GIST associated with limited peritoneal carcinomatosis. The kinase genotyping revealed a deletion mutation (c.1657_1674del p.Y553_K558del) in exon 11 of c-KIT. The patient underwent multidisciplinary treatment with surgery and several tyrosine kinase inhibitors. In December 2008, CT/PET revealed pelvic relapse. Two months later, a small nodular image in the right lower lobe was observed by CT evaluation, but was not suggestive of lung metastasis due to its small size (Fig. 2A). In July 2009, while the patient was being treated with sunitinib (Sutent ${ }^{\circledR}$, Pfizer, Inc., New York, NY, USA), a slight increase in lung nodule size was measured ( $9 \mathrm{~mm}$ compared to $5 \mathrm{~mm}$ ). In November 2009, a further increase (13 vs. 9 mm) was noted, concurrent with pelvic lesions (Fig. 2B and C). Therefore, the patient began third-line treatment with the novel agent nilotinib, (AMN107; Tasigna ${ }^{\circledR}$, Novartis Pharmaceuticals Corp.) and exhibited stable disease at the time of the most recent CT scan (Fig. 2D). 
A

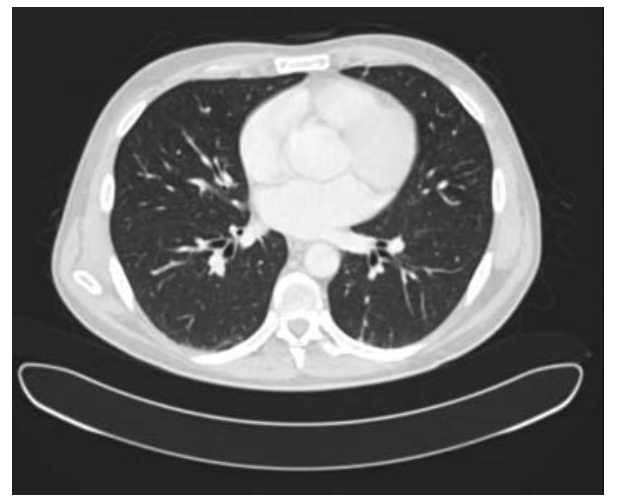

B
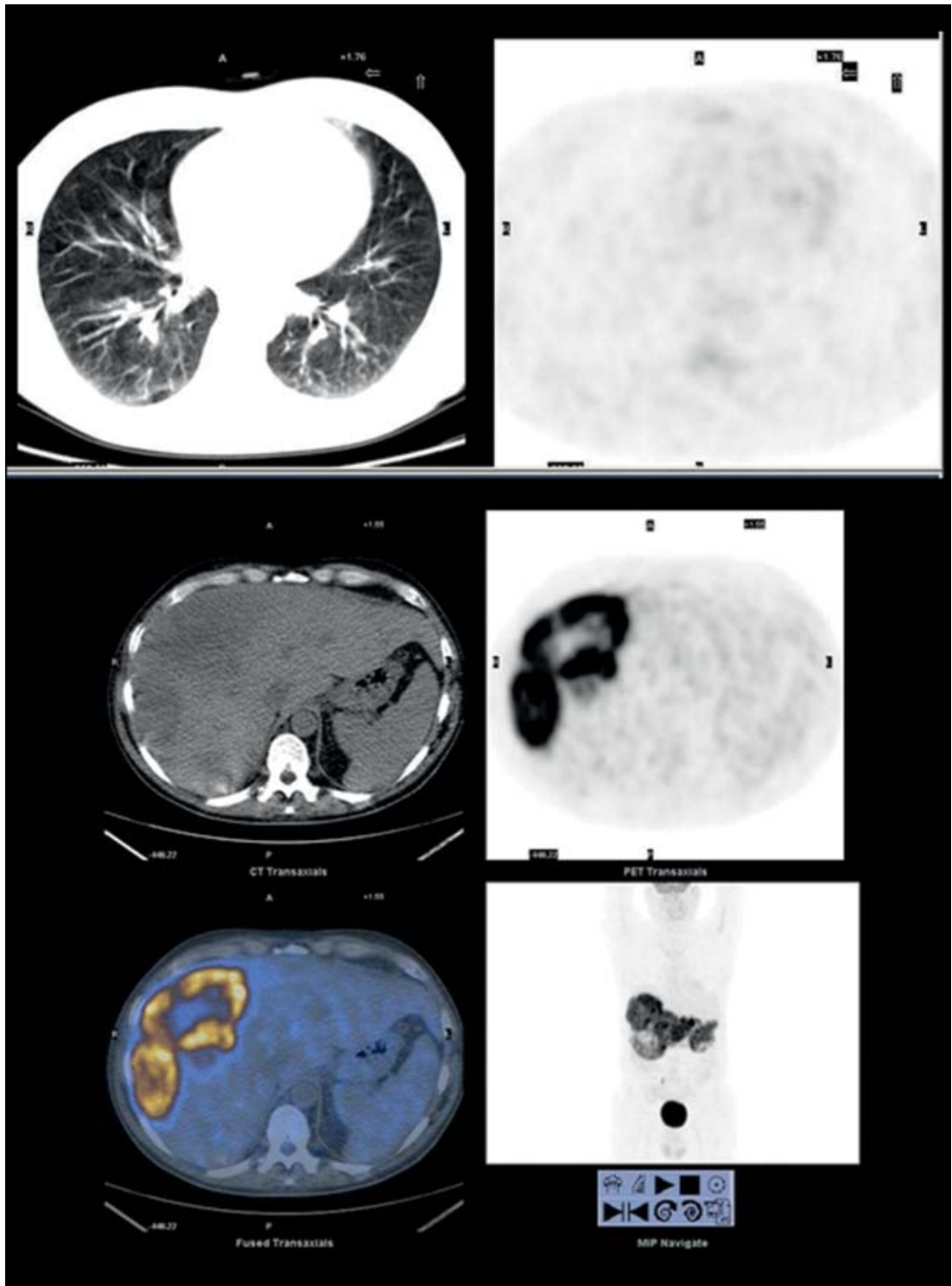

Figure 3. A and B. Computed tomography.

Case 3. In July 2008, a 44-year-old male underwent digiunal resection of a high-risk GIST. The kinase genotype revealed no c-KIT or PDGFRA mutations (WT). The patient was not treated with adjuvant imatinib because the disease was initially diagnosed as leiomyoma. In March 2009, a multifocal liver relapse occurred, and he underwent treatment with $400 \mathrm{mg}$ daily imatinib, which was increased to $800 \mathrm{mg}$ after disease progression. Six months later, CT revealed stable hepatic disease with a millimetric micronodule in the middle lobe, which was not believed to be pathologic due its small size (Fig. 3A). Subsequent CT/PET confirmed the metabolic stability of the hepatic lesions, which displayed no $18 \mathrm{~F}$ fluorodeoxyglucose (FDG) uptake in the pulmonary region (Fig. 3B). In November 2009, CT revealed an increased lung nodule diameter ( 6 vs. $2 \mathrm{~mm}$ ), but the CT/PET findings remained negative (Fig. 3C). Due to clinical disease progres- 
C

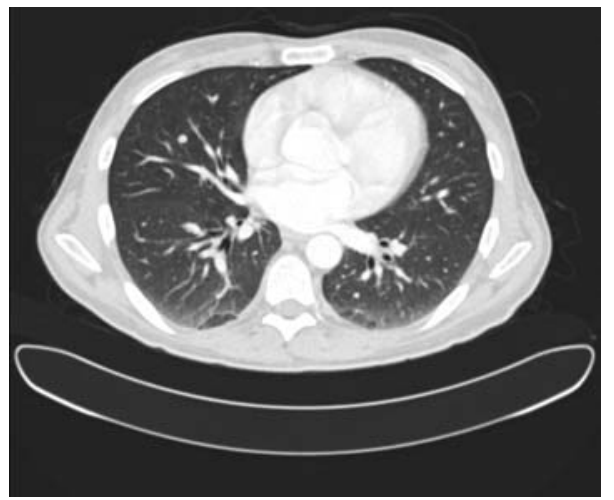

D

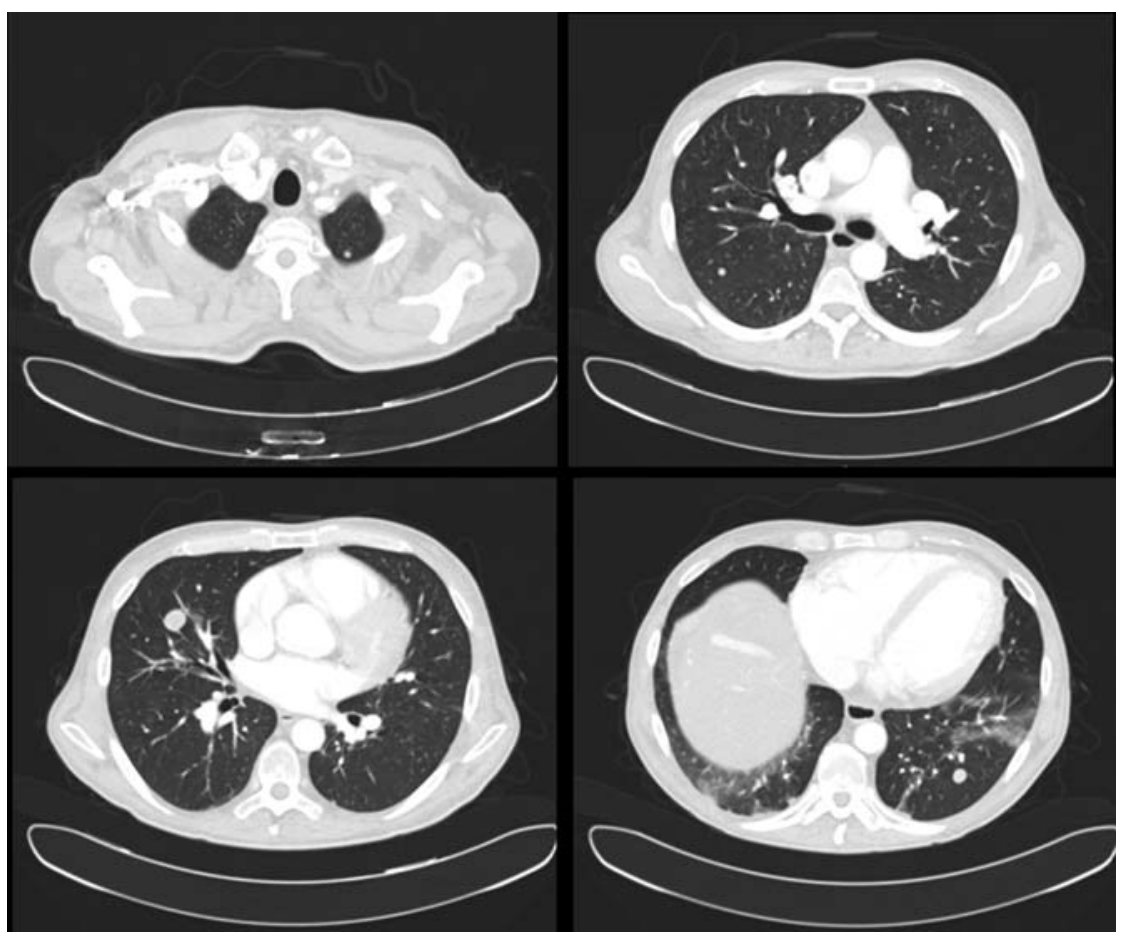

Figure 3. (A) Computed tomography (CT) taken in September 2009 revealed a micronodule at the middle lobe, which was not believed to be pathologic due to the small diameter of the tumor (2 mm). (B) September 2009: computed tomography/positron emission tomography (CT/PET) confirmed the metabolic stability of the hepatic lesions, revealing minimal 18F-fluorodeoxyglucose uptake in the pulmonary region. (C) November 2009: CT revealed an increase in the lung nodule diameter (6 vs. 2 mm). (D) March 2010: CT evaluation showed an increase in the number and diameter of the lung lesions.

sion, the patient began second-line therapy with $37.5 \mathrm{mg}$ daily sunitinib, but after three months of treatment the CT evaluation showed an increase in the number and diameter of the lung lesions (Fig. 3D). He is scheduled to begin third-line treatment with nilotinib.

Case 4. In 2005 a 28-year-old female was diagnosed with GIST of the stomach with liver and lung metastases, and was sequentially treated with imatinib and sunitinib. In 2007, she underwent total gastrectomy due to bleeding of the primary lesion. Histological examination confirmed that the metastases were WT and were derived from the primary lesion. The bilateral lung lesions and the liver metastases remained stable for five years, as monitored by CT and CT/ PET (Fig. 4A-C). The patient was being treated with nilotinib and exhibited stable disease at the most recent $\mathrm{CT}$ evaluation (Fig. 4C).

\section{Discussion}

The liver and peritoneum are the most frequent metastatic sites of gastrointestinal stromal tumors (GISTs) (6), but lung metastases from GISTs are relatively rare. The aim of the present case study was to describe the clinical, radiological, and molecular aspects of lung metastases arising from GISTs.

Clinically, lung metastases are asymptomatic due to their small size and intra-parenchymal distribution, and are frequently diagnosed as occasional findings. These lesions form as micronodules without initial pathologic significance, and may grow slowly (cases 2 and 3 ) or remain stable over longer periods (cases 1 and 4 ). In addition, these lung metastases do not appear to be associated with pathological mediastinal or hilar lymph nodes, as evidenced by the low frequency of GIST dissemination to the lymph nodes. Furthermore, there appeared to be no correlation between the 
A
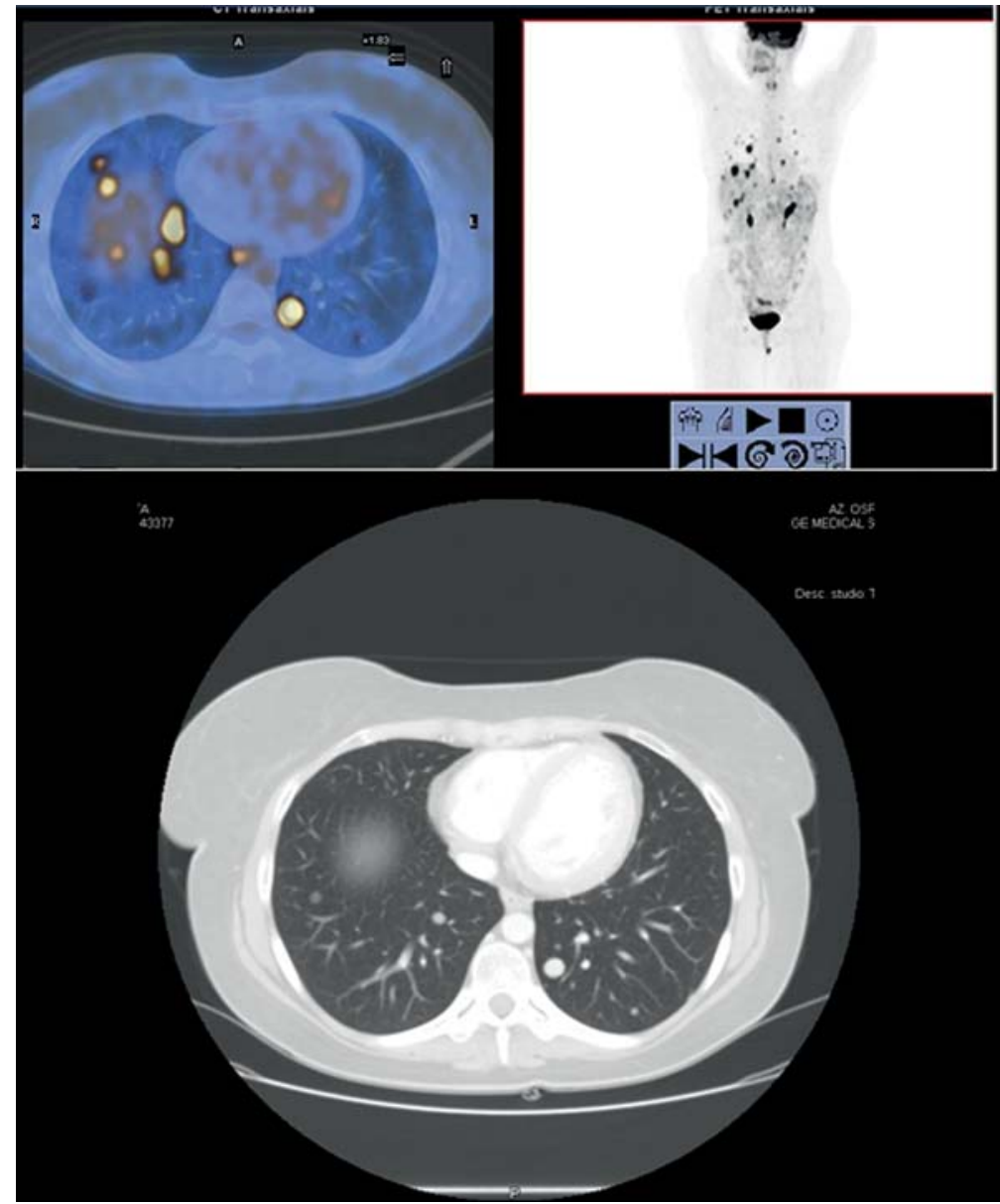

B
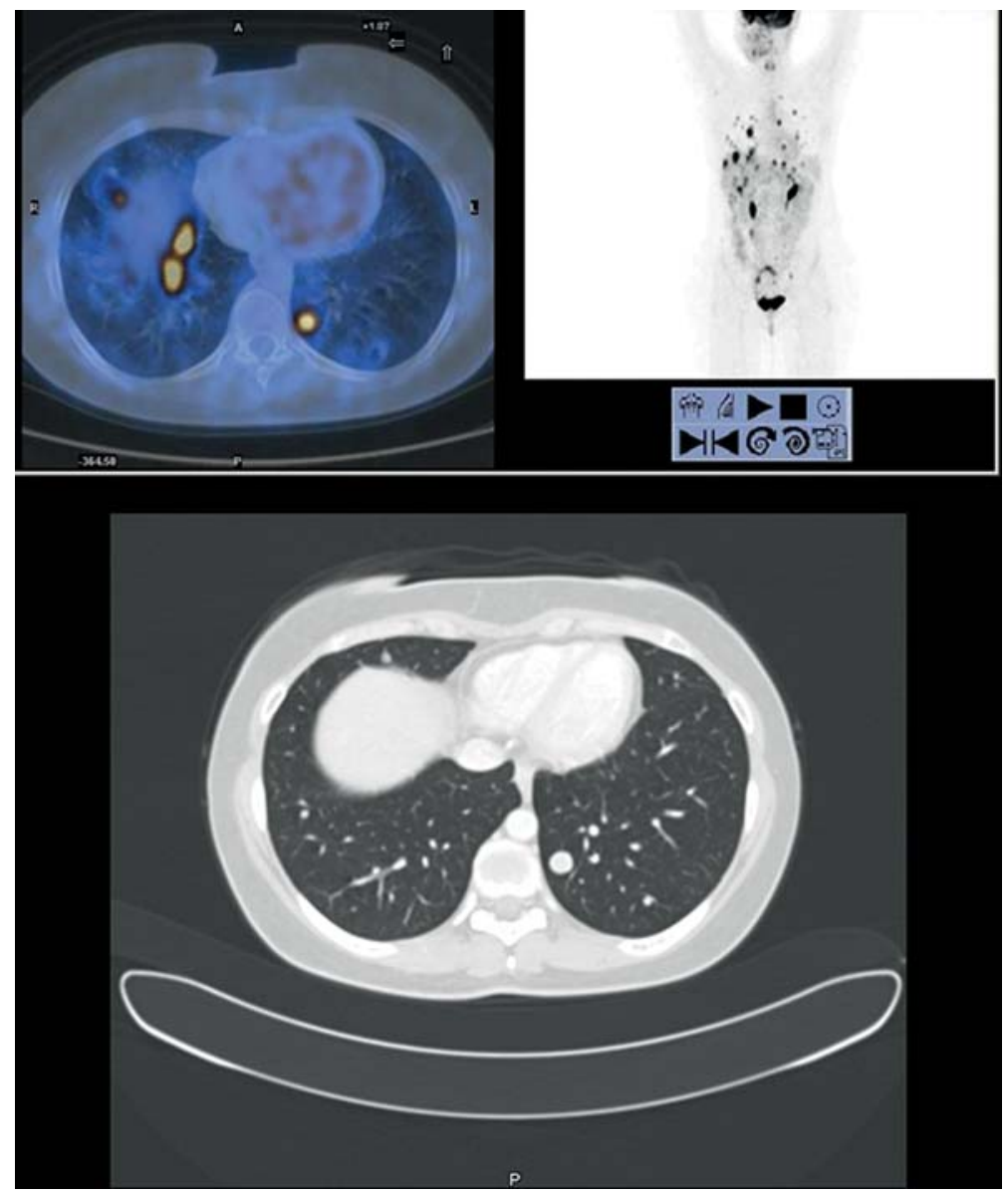

Figure 4. A and B. Bilateral lung lesions and liver metastases were observed at the time of patient diagnosis exhibiting positive computed tomography/ positron emission tomography. 


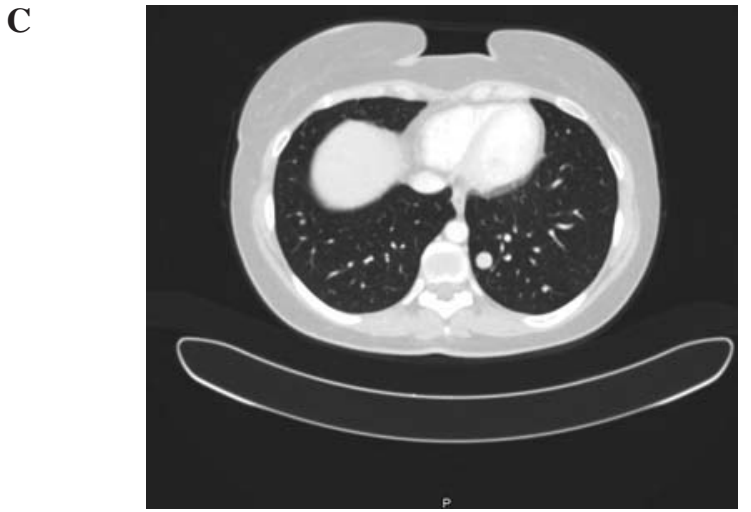

Figure 4. (A) Bilateral lung lesions and liver metastases were observed at the time of patient diagnosis exhibiting positive computed tomography/positron emission tomography (CT/PET) findings. (B) The lung lesions remained stable for five years as monitored by CT, and exhibited persistent CT/PET positivity, even throughout treatment. (C) At the time of the most recent CT evaluation, stable disease was observed.

risk of lung metastases and the site of the primary tumor, and lung metastases were frequently associated with other more common sites of relapse.

Radiologically, lung metastases have a well-defined CT pattern at the time of patient presentation. Lung lesions in all of the present patients were nodules characterized by a regular profile, were extremely hyperdense after contrast uptake, and had no intralesional areas of necrosis, even in patients with more a durable response to treatment. While the features of lung metastases were homogeneous on CT, CT/ PET findings varied between patients and between metastatic lesions in the same patient. CT/PET revealed FDG uptake in lung and liver lesions in patient 1 . However, patients 2 and 3 exhibited consistently negative FDG uptake in lung lesions and positive uptake by the liver metastases. This suggests that CT/PET scans may not be the optimal method to diagnose and stage GISTs $(13,14)$. The lack of FDG uptake may be related to the slow growth rate and small size of the lesions, suggesting that GIST lung metastases have a low intrinsic metabolism and a low biologic aggressiveness compared with the primary tumor or metastases at other sites. There is a positive relationship between FDG uptake and the Ki-67 proliferation index in non-small cell lung cancer, suggesting that differences in tumor cell proliferation are consistent with differences in tumor glucose metabolism (15). We found that the difference in FDG uptake between the primary tumor and the lung metastases (standardized uptake volume, $\mathrm{SUV}_{\text {max }}=55.1$ vs. 9.0, respectively) in patient 4 correlated with the difference in the Ki-67 proliferation index for these two lesions (12.5 and 5\%, respectively). Because of potential limitations of FDG-CT/PET for the diagnosis of GIST lung metastases due to variations in tumor size or biological limits, other PET tracers that specifically target cell proliferation, such as $18 \mathrm{~F}$-fluorodeoxythymidine (FLT), may have higher diagnostic sensitivity (16).

Lung metastases appear to respond differently to treatment than liver and peritoneal metastases, regardless of primary tumor c-KIT- and PDGFRA molecular status. The radiological hallmark of the metastatic lesions after tyrosine kinase inhibitors in our patients was hyperdensity without necrotic areas. Patient 1 had a pulmonary partial response after three months of treatment with imatinib, but the lung lesion was reduced in size and maintained a consistent radio- logical pattern without the typical signs of intralesional tumor response. This finding is inconsistent with the consolidated concept of response evaluation to target therapies. Noncytotoxic drugs do not initially cause reduction in tumor volume, and in fact can lead to a paradoxical size increase due to necrosis. Therefore, tumor response evaluation is based both on intralesional density variation and changes in tumor diameter. The lack of observed density variation is inconsistent with the Choi criteria for the evaluation of response to TKinhibitors $(17,18)$. Patient 1 exhibited predictable CT/PET density findings during imatinib therapy due to reduced FDG uptake, whereas patient 4 showed a longer-term positivity of lung metastases, even during treatment. In general, lung metastases were more stable throughout tyrosine kinase inhibitor treatment, which was most likely due to the indolent behavior of these tumors. We do not yet have sufficient data to correlate KIT and PDGFRA genotypes to clinical responses to tyrosine kinase inhibitors. Two patients had a primary GIST with a WT mutational status, and the remaining two patients exhibited c-KIT exon 11 mutations. Kinase genotyping could only be performed in patient 4 , whose primary and metastatic tumors were classified as WT for KIT and PDGFRA.

Recently, the presence of lung metastases in the absence of liver metastases was suggested to be an independent predictor of initial resistance to imatinib in patients with GIST (19). However, lung metastases did not affect the initial tumor response in patients with an external confirmation of GIST diagnosis, with the exception of misdiagnosed sarcomas that are notoriously unresponsive to imatinib (19). Although none of our patients presented with GIST metastases only to the lung, the small number of patients, the long natural history (cases 2 and 4), and the WT molecular profile (cases 3 and 4) may explain the limited clinical response to standard tyrosine kinase inhibitors. No conclusions may be drawn regarding secondary resistance, which appears to be a late event. The management of GIST lung metastases remains difficult because of multiple, bilateral and other associated non-resectable metastases. However, the low aggressiveness and lack of concomitant lymphatic spread suggest that surgery or other loco-regional treatments could be considered in cases of lesions confined to the lung, or in cases of resectable and responsive metastatic disease. 
Although lung metastases are relatively rare in patients with GIST, they may become more prevalent due to the increase in patient life expectancy. It remains unclear whether there is a specific molecular profile that can predict whether a patient is at risk for these lung metastases. Novel molecular technologies and larger retrospective multicenter studies are needed to determine the best treatment for patients with lung metastases derived from primary GISTs.

\section{References}

1. Corless CL, Fletcher JA and Heinrich MC: Biology of gastrointestinal stromal tumors. J Clin Oncol 22: 3813-3825, 2004.

2. Hirota S, Isozaki K, Moriyama Y, Hashimoto K, Nishida T, Ishiguro S, Kawano K, Hanada M, Kurata A, Takeda M, Muhammad Tunio G, Matsuzawa Y, Kanakura Y, Shinomura Y and Kitamura Y: Gain-of-function mutations of c-kit in human gastrointestinal stromal tumors. Science 279: 577-580, 1998.

3. Heinrich MC, Corless CL, Duensing A, McGreevey L, Chen CJ, Joseph N, Singer S, Griffith DJ, Haley A, Town A, Demetri GD, Fletcher CD and Fletcher JA: PDGFRA activating mutations in gastrointestinal stromal tumors. Science 299: 708-710, 2003.

4. Heinrich MC, Corless CL, Demetri GD, Blanke CD, von Mehren M, Joensuu H, McGreevey LS, Chen CJ, van den Abbeele AD, Druker BJ, Kiese B, Eisenberg B, Roberts PJ, Singer S, Fletcher CD, Silberman S, Dimitrijevic S and Fletcher JA: Kinase mutations and imatinib response in patients with metastatic gastrointestinal stromal tumors. J Clin Oncol 21: 4342-4349, 2003.

5. Heinrich MC, Maki RG, Corless CL, Antonescu CR, Harlow A, Griffith D, Town A, McKinley A, Ou WB, Fletcher JA, Fletcher CD, Huang X, Cohen DP, Baum CM and Demetri GD: Primary and secondary kinase genotypes correlate with the biological and clinical activity of sunitinib in imatinib-resistant gastrointestinal stromal tumor. J Clin Oncol 33: 5352-5359, 2008 .

6. DeMatteo RP, Lewis JJ, Leung D, Mudan SS, Woodruff JM and Brennan MF: Two hundred gastrointestinal stromal tumors: recurrence patterns and prognostic factors for survival. Ann Surg 231: 51-58, 2000.

7. Inage Y, Yamabe K, Yamamoto T, Sato Y, Ishikawa S, Onizuka M and Yoshida K: Resection for pulmonary metastasis of gastrointestinal stromal tumor of the stomach at 10 years after gastrectomy; report of a case. Kyobu Geka 55: 907-911, 2002 .

8. Catani M, De Milito R and Simi M: New orientations in the management of advanced, metastatic gastrointestinal stromal tumors (GIST): combination of surgery and systemic therapy with imatinib in a case of primary gastric location. Chir Ital 57: 127-133, 2005.
9. De Chiara A, De Rosa V, Lastoria S, Franco R, Botti G, Iaffaioli VR and Apice G: Primary gastrointestinal stromal tumor of the liver with lung metastases successfully treated with STI-571 (imatinib mesylate). Front Biosci 11: 498-501, 2006.

10. Ozan E, Oztekin O, Alacacioglu A, Aykas A, Postaci H and Adibelli Z: Esophageal gastrointestinal stromal tumor with pulmonary and bone metastases. Diagn Interv Radiol 16: 217-220, 2010.

11. Kurashina K, Hosoya Y, Sakurai S, Endo S, Lefor A and Yasuda Y: Gastrointestinal stromal tumor of the stomach with extremely slow-growing hematogenous metastasis. Int J Clin Oncol 14: 262-265, 2009.

12. Bertulli R, Fumagalli E, Coco P, Messina A, Morosi C, Dileo P and Casali PG: Unusual metastatic sites in gastrointestinal stromal tumor (GIST). J Clin Oncol 27: abs. 10566, 2009.

13. Gayed I, Vu T, Iyer R, Johnson M, Macapinlac H, Swanston N and Podoloff D: The role of 18F-FDG PET in staging and early prediction of response to therapy of recurrent gastrointestinal stromal tumors. J Nucl Med 45: 17-21, 2004.

14. Van den Abbeele AD: The lessons of GIST-PET and PET/CT: a new paradigm for imaging. Oncologist 13: 8-13, 2008.

15. Vesselle H, Salskov A, Turcotte E, Wiens L, Schmidt R, Jordan CD, Vallières E and Wood DE: Relationship between non-small cell lung cancer FDG uptake at PET, tumor histology, and Ki-67 proliferation index. J Thorac Oncol 3: 971-978, 2008.

16. Pantaleo MA, Landuzzi L, Nicoletti G, Nanni C, Boschi S, Piazzi G, Santini D, Di Battista M, Castellucci P, Lodi F, Fanti S, Lollini PL and Biasco G: Advances in preclinical therapeutics development using small animal imaging and molecular analyses: the gastrointestinal stromal tumors model. Clin Exp Med 9: 199-205, 2009.

17. Choi H, Charnsangavej C, Faria SC, Macapinlac HA, Burgess MA, Patel SR, Chen LL, Podoloff DA and Benjamin RS: Correlation of computed tomography and positron emission tomography in patients with metastatic gastrointestinal stromal tumor treated at a single institution with imatinib mesylate: proposal of new computed tomography response criteria. J Clin Oncol 25: 1753-1759, 2007.

18. Benjamin RS, Choi H, Macapinlac HA, Burgess MA, Patel SR, Chen LL, Podoloff DA and Charnsangavej C: We should desist using RECIST, at least in GIST. J Clin Oncol 25: 1760-1764, 2007.

19. Van Glabbeke M, Verweij J, Casali PG, Le Cesne A, Hohenberger P, Ray-Coquard I, Schlemmer M, van Oosterom AT, Goldstein D, Sciot R, Hogendoorn PC, Brown M, Bertulli R and Judson IR: Initial and late resistance to imatinib in advanced gastrointestinal stromal tumors are predicted by different prognostic factors: a European Organisation for Research and Treatment of Cancer-Italian Sarcoma Group-Australasian Gastrointestinal Trials Group study. J Clin Oncol 23: 5795-5804, 2005. 